\title{
Symposium review: The uterine microbiome associated with the development of uterine disease in dairy cows*
}

\author{
Klibs N. Galvão, ${ }^{1,2} \dagger$ ๑ Rodrigo C. Bicalho, ${ }^{3} \oplus$ and Soo Jin Jeon ${ }^{4}$ \\ ${ }^{1}$ Department of Large Animal Clinical Sciences, College of Veterinary Medicine, University of Florida, Gainesville 32610 \\ ${ }^{2}$ D. H. Barron Reproductive and Perinatal Biology Research Program, University of Florida, Gainesville 32610 \\ ${ }^{3}$ Department of Population Medicine and Diagnostic Sciences, Cornell University, Ithaca, NY 14853 \\ ${ }^{4}$ Department of Biomedical Veterinary Sciences, College of Veterinary Medicine, Long Island University, Brookville, NY 11548
}

\section{ABSTRACT}

Until 2010, our knowledge of the uterine microbiome in cows that developed uterine disease relied almost exclusively on culture-dependent studies and mostly included cows with clinical endometritis (i.e., with purulent uterine discharge). Those studies consistently found a strong positive correlation between Trueperella pyogenes and clinical endometritis, whereas other pathogens such as Escherichia coli, Fusobacterium necrophorum, Prevotella melaninogenica, and Bacteroides spp. were also commonly cocultured. In contrast, Streptococcus spp., Staphylococcus spp., and Bacillus spp. were usually isolated from healthy cows. Starting in 2010, culture-independent studies using PCR explored the microbiome of cows with metritis and clinical endometritis, and observed that E. coli was a pioneer pathogen that predisposed cows to infection with $F$. necrophorum, which was strongly associated with metritis, and to infection with T. pyogenes, which was strongly associated with clinical endometritis. Starting in 2011, culture-independent studies using metagenomic sequencing expanded our knowledge of the uterine microbiome. It has been shown that cows have bacteria in the uterus even before calving, they have an established uterine microbiome within 20 min of calving, and that the microbiome structure is identical between cows that develop metritis and healthy cows until $2 \mathrm{~d}$ postpartum, after which the bacterial structure of cows that developed metritis deviates in favor of greater relative abundance of Bacteroidetes and Fusobacteria and lesser relative abundance of Proteobacteria and Tenericutes. The shift in the uterine microbiome in cows that develop metritis is characterized by a loss of heterogeneity and a decrease in bacterial richness. At the genus level,

\footnotetext{
Received June 13, 2019.

Accepted August 22, 2019.

*Presented as part of the Reproduction Platform Session: Transition Cow Health and Reproduction at the ADSA Annual Meeting, Cincinnati, Ohio, June 2019

†Corresponding author: galvaok@ufl.edu
}

Bacteroides, Porphyromonas, and Fusobacterium have the strongest association with metritis. At the species level, we observed that Bacteroides pyogenes, Porphyromonas levii, and Helcococcus ovis were potential emerging uterine pathogens. Finally, we have shown that the hematogenous route is a viable route of uterine infection with uterine pathogens. Herein, we propose that metritis is associated with a dysbiosis of the uterine microbiota characterized by decreased richness, and an increase in Bacteroidetes and Fusobacteria, particularly Bacteroides, Porphyromonas, and Fusobacterium. Key words: metritis, uterine microbiota, dairy cow

\section{INTRODUCTION}

The transition to lactation is a challenging period for a high-producing dairy cow. This period is characterized by a sharp decrease in immune function (Kehrli and Goff, 1989; Cai et al., 1994). At the same time, physical barriers such as the cervix are breached at parturition, which allows for rapid colonization of the uterus by bacteria (Elliott et al., 1968; Sheldon and Dobson, 2004). Cows defend themselves against invading pathogens using tolerance and resistance mechanisms. Tolerance is the ability to limit the disease severity induced by a given pathogen burden, and there is evidence that high milk yield impairs disease tolerance (Sheldon et al., 2019). Resistance is the ability to limit the pathogen burden and is usually a function of immunity. However, the system is overwhelmed by a decrease in immune function, a large bacterial challenge, and reduced tolerance in high-producing dairy cows, and uterine diseases such as metritis, clinical endometritis, and subclinical endometritis are established in a large proportion of cows in early postpartum. Metritis and clinical endometritis have a prevalence of $\sim 20 \%$, ranging from $8 \%$ to $>40 \%$ in some farms (LeBlanc, 2008; Galvão, 2012). We recently completed a survey of 16 farms in 6 regions of the United States, in which 11,733 cows were checked within the first $14 \mathrm{~d}$ postpartum (DPP) and 11,129 were checked from 30 to $36 \mathrm{DPP}$. 
We observed a prevalence of metritis of $25 \%$ (range: 14 to $44 \%$ ) and a prevalence of clinical endometritis of 26\% (range: 12 to 35\%; Gonzalez-Pena et al., 2016). Of the cows evaluated for both diseases, $29.3 \%$ had one or the other and $10.6 \%$ had both; therefore, $39.9 \%$ of the cows had uterine disease postpartum. Numerous studies have demonstrated both direct and indirect negative effects of metritis on overall dairy herd performance and profitability (Goshen and Shpigel, 2006; Overton and Fetrow, 2008).

The causes of uterine disease are multifactorial. Of the 3 components of the disease triangle, there is good evidence for the susceptibility of the host and the role of pathogenic bacteria but less evidence for the effect of the environment (Sheldon and Dobson, 2004), although it cannot be disregarded (Schuenemann et al., 2011). The major risk factors for uterine disease development, the role of the host immune response in the development of uterine diseases, and the consequences of uterine disease have been previously reviewed (Sheldon et al., 2009; Galvão and Santos, 2014; Sheldon et al., 2019). Hence, this review will focus on the role of the uterine microbiome in the development of metritis in dairy cows. We pay special attention to recent advances in the use of metagenomic sequencing to characterize the uterine microbiome of cows that develop metritis, a comparison of the uterine microbiome in cows with and without a fever at the time of metritis diagnosis, the progression of the uterine microbiome after metritis diagnosis, particularly in cows that cure and fail to cure metritis, and the establishment of hematogenous route as a possible route of uterine infection with uterine pathogens.

\section{UTERINE MICROBIOME IN COWS THAT DEVELOP METRITIS: CULTURE-DEPENDENT STUDIES}

Until 2010, our knowledge of the postpartum uterine microbiome in dairy cows that developed uterine disease relied almost exclusively on culture-dependent studies (Griffin et al., 1974; Studer and Morrow, 1978; Ruder et al., 1981; Olson et al., 1984; Bonnett et al., 1991; Noakes et al., 1991; Dohmen et al., 1995; Huszenicza et al., 1999; Dohmen et al., 2000; Williams et al., 2005). Moreover, most studies evaluated cows with clinical endometritis (i.e., presence of pus in the uterine discharge) or pyometra (i.e., presence of pus in uterus in the presence of a corpus luteum), with the exceptions being Huszenicza et al. (1999) and Drillich et al. (2001), who evaluated cows with metritis within 14 DPP. Thus, the caveat is that a clear definition of metritis and clinical endometritis only became available in the early 2000s (LeBlanc et al., 2002a; Sheldon et al., 2006). Based on the previous literature, Sheldon et al. (2002) classified bacteria according to their expected pathogenicity, and they considered Trueperella (formerly Arcanobacterium) pyogenes, Escherichia coli, Prevotella melaninogenica, and Fusobacterium necrophorum as recognized uterine pathogens, although the literature was not always consistent on the associations of these 4 pathogens with uterine disease, particularly in regards to the involvement of E. coli and Prev. melaninogenica. Nonetheless, this classification was widely disseminated and became the accepted dogma (Sheldon and Dobson, 2004; Williams et al., 2005; Sheldon et al., 2009).

Williams et al. (2005) sampled cows from 21 to 28 DPP and reported that T. pyogenes and E. coli were the most prevalent recognized uterine pathogens isolated from the uterus, followed by Prev. melaninogenica and F. necrophorum. They observed that cows diagnosed with endometritis (i.e., purulent vaginal discharge) had greater growth density of recognized and potential uterine pathogens but not of opportunists. Cows with T. pyogenes were more likely to be diagnosed with endometritis and more likely to have fetid vaginal mucus. Cows with $E$. coli were more likely to have fetid vaginal mucus. Cows with $F$. necrophorum were more likely to be diagnosed with mucopurulent vaginal discharge but not purulent vaginal discharge; Prev. melaninogenica was not associated with mucopurulent or purulent vaginal discharge.

Trueperella pyogenes has long been known capable of causing endometritis in animals (Lamming et al., 1955). Reviewing the previous literature, we found that Griffin et al. (1974) were among the first to report the association of $T$. pyogenes with endometrial inflammation and infertility in cows. Others (Studer and Morrow, 1978; Bonnett et al., 1991) later confirmed the association between isolation of T. pyogenes and endometrial inflammation. Studer and Morrow (1978) also reported that cows with $T$. pyogenes were more likely to be diagnosed with clinical endometritis (i.e., purulent vaginal discharge) and had the most severe histological lesions. They also reported that coliforms (E. coli or Aerobacter spp.) were associated with clinical endometritis and endometrial histological changes. In contrast, Bonnett et al. (1991) reported no association between E. coli and endometrial inflammation. Dohmen et al. (1995) reported that T. pyogenes, F. necrophorum, and Bacteroidetes, particularly Porphyromonas levii but also Prev. melaninogenica, were correlated among themselves, and were isolated with higher frequency as the uterine discharge score worsened (i.e., from flecks of pus to hemorrhagic and fetid). Previous studies commonly reported the isolation of $F$. necrophorum in combination with T. pyogenes (Ruder et al., 1981; Olson et al., 1984; Noakes et al., 1991; Dohmen et al., 
1995) and other bacteria such as Prev. melaninogenica (Olson et al., 1984; Noakes et al., 1991) from cows with endometritis or pyometra. Hence, based on the findings by Olson et al. (1984) and Noakes et al. (1991), T. pyogenes. F. necrophorum, and Prev. melaninogenica alone or in combination were used to induce pyometra in dairy cows (Farin et al., 1989), which showed that these bacteria can cause uterine disease. More recent studies have shown that infusion of $T$. pyogenes alone (Lima et al., 2015) or in combination with E. coli (Piersanti et al., 2019) can cause uterine inflammation.

Huszenicza et al. (1999) reported findings similar to Williams et al. (2005) in cows with metritis; namely, a greater prevalence of $T$. pyogenes and E. coli in cows with metritis. Additionally, they reported that cows with metritis had greater prevalence of gram-negative anaerobes, particularly Bacteroides spp., but interestingly, they reported a low prevalence of $F$. necrophorum and black-pigmented Bacteroides such as Por. levii and Prev. melaninogenica. In contrast, Drillich et al. (2001) sampled 15 cows with metritis and reported that $F$. necrophorum and Por. levii were the most prevalent (67\%) bacteria isolated. Interestingly, the role of Por. levii in the development of uterine disease has been largely overlooked (Sheldon et al., 2002; Williams et al., 2005).

In contrast to observations by Williams et al. (2005), Dohmen et al. (1995) observed that E. coli was isolated less frequently as uterine discharge worsened. Furthermore, E. coli was negatively associated with the presence of T. pyogenes at 14 DPP. Bonnett et al. (1993) reported that cows with $T$. pyogenes at $40 \mathrm{DPP}$ had poor reproductive performance, which was later confirmed by others (Huszenicza et al., 1999; Gilbert and Santos, 2016). Bonnett et al. (1993) also reported that cows with $E$. coli tended to have good reproductive performance (i.e., conceived by 119 DPP), which supports the findings by Dohmen et al. (1995). de Boer et al. (2015) reported that cows with $E$. coli at calving were more likely to have $E$. coli or any other bacteria at 21 DPP and to be diagnosed with endometritis at 21 DPP. Ultimately, cows with $E$. coli at 21 DPP tended to take longer to conceive after the start of the breeding season (de Boer et al., 2015). Others have reported that cows with $E$. coli in the first week postpartum were more likely to have $T$. pyogenes and gram-negative anaerobes (i.e., F. necrophorum and Bacteroides spp.) in the second (Dohmen et al., 2000) and third (Gilbert and Santos, 2016) weeks postpartum. Therefore, although the association between E. coli and uterine disease and fertility has been conflicting, the findings by Dohmen et al. (2000), de Boer et al. (2015), and Gilbert and Santos (2016) support a long-held view that E. coli is a pioneer organism that sets up the environment for other pathogens such as T. pyogenes and gram-negative anaerobes to become established (Sheldon and Dobson, 2004).

In summary, the results from culture-dependent studies indicate that $T$. pyogenes is a critical pathogen involved in the development of clinical endometritis, and that gram-negative anaerobes such as $F$. necrophorum, Por. levii, and Prev. melaninogenica may act synergistically with $T$. pyogenes to cause clinical endometritis. Culture-dependent studies also support the involvement of $E$. coli either as a main pathogen or as a pioneer pathogen that sets up stage for T. pyogenes and gramnegative anaerobes. Although data were scarcer for cows with metritis, the available data also point to an involvement of $T$. pyogenes, E. coli, and gram-negative anaerobes in the development of metritis.

In regards to bacteria associated with uterine health, Bonnett et al. (1991) reported that isolation of $\alpha$-hemolytic streptococci was negatively correlated with isolation of $A$. pyogenes and with inflammation. Dohmen et al. (1995) reported that streptococci were isolated less frequently as the uterine discharge worsened, and that streptococci were negatively associated with the presence of $T$. pyogenes at 14 DPP. Williams et al. (2005) observed that cows with $\alpha$-hemolytic streptococci, the most common opportunist, and coagulase-negative staphylococci were less likely to be diagnosed with endometritis. Huszenicza et al. (1999) also reported a lower prevalence of streptococci and staphylococci in cows with metritis compared with healthy cows. Gilbert and Santos (2016) reported that cows with $\alpha$-hemolytic streptococci at 7 DPP had improved fertility. Therefore, culture-based studies indicate that streptococci, or more specifically, $\alpha$-hemolytic streptococci, can promote uterine health. There were also some indications that E. coli detected later in lactation could promote uterine health (Bonnett et al., 1993; Dohmen et al., 1995) but these findings were inconsistent (de Boer et al., 2015).

\section{UTERINE MICROBIOME IN COWS THAT DEVELOP METRITIS: CULTURE-INDEPENDENT STUDIES}

\section{Study of the Uterine Microbiome Using PCR-Based Techniques}

Culture-dependent methods have an inherent limitation of only being able to detect bacteria for which a culturing and identification method has been established. Indeed, it has been estimated that less than $1 \%$ of bacteria can be cultured in the laboratory (Kaeberlein et al., 2002). To overcome this limitation, culture-independent techniques such as PCR, clone library sequencing, 16S rRNA metagenomic sequencing, 
and whole-metagenome sequencing have been used to elucidate the role of the main uterine pathogens identified in culture-dependent studies. Nonetheless, these techniques are not without drawbacks. Limitations include the following: (1) bacterial viability cannot be evaluated; (2) sequencing depth and coverage are low when using clone library sequencing; (3) operational taxonomic unit classification may change depending on the variable region of the $16 \mathrm{~S}$ rRNA gene that is targeted for sequencing; (4) classification based on $16 \mathrm{~S}$ rRNA gene sequencing is not accurate at the species level; and (5) cost, which tends to be higher, particularly for whole metagenome sequencing (Chakravorty et al., 2007). The choice of variable region of the $16 \mathrm{~S}$ rRNA gene to be targeted has been extensively studied because it may affect taxonomic classification and diversity estimates. Nonetheless, different studies have arrived at different conclusions, with recommendations including one or more of the V2, V3, V4, V6, or V3/V4 regions (Mizrahi-Man et al., 2013).

Starting in 2010, several studies using culture-independent techniques helped us expand our understanding of the uterine microbiome associated with uterine disease. Using multilocus sequence typing, Sheldon et al. (2010) identified specific strains of $E$. coli from animals with endometritis. These strains were more adherent and invasive to endometrial epithelial and stromal cells than E. coli isolated from healthy cows. Although they could not identify a specific virulence factor responsible for this increased adherence and invasion, a mannose-binding assay pointed to the involvement of $\mathrm{fimH}$. The $\mathrm{fimH}$ gene is expressed in more than $90 \%$ of uropathogenic E. coli and encodes type 1 fimbrial D-mannose specific adhesion, which is central to its pathogenicity in the urogenital tract of women (Tchesnokova et al., 2011). Bicalho et al. (2010) screened for the presence of 32 virulence factors in $E$. coli isolated from the uterus of dairy cows within the first week postpartum and observed that prevalence of metritis was higher in cows with $E$. coli possessing fimH alone or in combination with other virulence factors such as $h l y A, c d t, k p s M I I, i b e A$, or astA. Later, the same group observed that cows positive for fimH at 1 to $3 \mathrm{DPP}$ were more likely to be diagnosed with metritis at 8 to $10 \mathrm{DPP}$ and with endometritis at 34 to $35 \mathrm{DPP}$ (Bicalho et al., 2012). Cows positive for fimH at 1 to 3 DPP were also more likely to be positive for $F$. necrophorum lktA at 8 to $10 \mathrm{DPP}$, which increased the likelihood of having metritis at 8 to 10 DPP. Furthermore, cows positive for F. necrophorum lktA at 34 to 36 DPP were also more likely to be positive for T. pyogenes fimA, which in turn had the greatest positive correlation with clinical endometritis at 34 to $36 \mathrm{DPP}$. Ultimately, cows positive for fim $H$ at 1 to 3 DPP had decreased hazard of pregnancy. Interestingly, being positive for E. coli or T. pyogenes at 8 to $10 \mathrm{DPP}$ was not associated with a diagnosis of metritis at 8 to $10 \mathrm{DPP}$. We have observed that abundance of E. coli and T. pyogenes at the time of metritis diagnosis, determined by quantitative PCR, was low and not different between metritic and healthy cows, whereas metritic cows had greater abundance of F. necrophorum, Por. levii, and Prev. melaninogenica compared with healthy cows (Cunha et al., 2018). Furthermore, being positive for $E$. coli at 34 to $36 \mathrm{DPP}$ was not associated with a diagnosis of clinical endometritis at 34 to $36 \mathrm{DPP}$. These findings support the idea of $E$. coli being a pioneer pathogen and highlight the synergism between E. coli, F. necrophorum, and T. pyogenes in causing metritis and clinical endometritis (Bicalho et al., 2012). Therefore, our interpretation of these data is that $E$. coli predisposes to uterine colonization by $F$. necrophorum, which is the main pathogen associated with the development of metritis, and F. necrophorum predisposes to colonization by $T$. pyogenes, which act synergistically with $F$. necrophorum to cause clinical endometritis.

\section{Study of the Uterine Microbiome Using Metagenomic DNA Sequencing Techniques}

Although PCR is a great tool to investigate specific pathogens, it provides only a narrow view of the whole microbial community. Therefore, to overcome this limitation, recent studies have used metagenomic sequencing to identify the bacterial community of cows with and without uterine disease (Santos et al., 2011; Machado et al., 2012; Jeon et al., 2015). Using clone library sequencing of composite samples from 2 farms, Santos et al. (2011) observed higher prevalence of Fusobacteria (particularly F. necrophorum) and Bacteroidetes (particularly Por. levii) in metritic cows and higher prevalence of Proteobacteria and Tenericutes in healthy cows. Interestingly, neither $E$. coli nor $T$. pyogenes sequences were detected in any of the 4 libraries. This was the first study using metagenomic sequences that pointed to the importance of Fusobacteria and Bacteroidetes at the time of metritis diagnosis, confirmed the importance of $F$. necrophorum, raised the profile of Por. levii as an emerging pathogen, and questioned the relevance of E. coli and T. pyogenes at the time of metritis diagnosis. Nonetheless, this study had limitations, including the use of composite samples, the lack of longitudinal sampling, and the use of clone library sequencing, which has a low depth of coverage.

To overcome some of the limitations in the study by Santos et al. (2011), we used 16S rRNA gene sequencing to characterize the uterine microbiota progression from calving until establishment of metritis in dairy cows 
(Jeon et al., 2015). We observed 28 phyla and 824 genera of bacteria in our uterine samples. We showed that all cows had an established uterine microbiota within 20 min of calving, and that the uterine microbiome changed rapidly from calving to $6 \pm 2$ DPP (Figure $1 \mathrm{~A}, 1 \mathrm{~B})$ with a reduction in the relative abundance of Proteobacteria (particularly Coxiella) and an increase in the relative abundance of Bacteroidetes (particularly Bacteroides and Porphyromonas) and Fusobacteria (particularly Fusobacterium; Jeon et al., 2015). Additionally, we showed that the uterine microbiome structure was identical between cows that later developed metritis and healthy cows until 2 DPP, after which the bacterial structure deviated in favor of greater relative abundance of Bacteroidetes, particularly Bacteroides and Porphyromonas (Jeon et al., 2015). This dysbiosis in the microbial community in metritic cows was characterized by consolidation of the uterine microbiome (Figure 2A-F) and a loss of microbial richness and diversity (Jeon et al., 2015, 2016), while at the same time the total bacterial load was increased (Figure 1C). Similar dynamics in bacterial load have been observed by others (Bicalho et al., 2017b).

Interestingly, the relative abundance of Fusobacteria was similar between healthy and metritic cows (Jeon et al., 2015). Nonetheless, at the genus level, discriminant analysis, correlation among bacteria, and correlation between bacteria and uterine discharge score pointed to a synergism among Bacteroides, Porphyromonas, and Fusobacterium in the development of metritis (Jeon et al., 2015). We also observed that failure to cure metritis was associated with an increase in the relative abundance of Bacteroides, Porphyromonas, and Fusobacterium, and a further decrease in bacterial diversity (Jeon et al., 2018). Later, we observed that the microbiome of metritic cows with or without a fever was virtu- ally identical, and that independent of the presence of fever at the time of diagnosis, metritic cows had greater prevalence of Bacteroides and Porphyromonas and numerically greater prevalence of Fusobacterium (Jeon et al., 2016). Greater abundance of Bacteroides, Porphyromonas, or Fusobacterium in metritic cows has since been observed by others (Knudsen et al., 2016; Bicalho et al., 2017a; Sicsic et al., 2018; Galvão et al., 2019), but for this review, we compiled a large data set with 80 metritic and 31 healthy cows to be able to infer with more certainty what pathogens are associated with metritis at the time of diagnosis. Using this data set, we performed linear discriminant analysis effect size (LEfSe) to determine the phyla and genera most likely to explain differences between healthy and metritic cows (Segata et al., 2011), in addition to ordination using non-metric multidimensional scaling (Figure 3AD). With this large data set, we confirmed the loss of richness and consolidation of the uterine microbiome (Figure 3A,B) observed in our previous studies (Jeon et al., 2015, 2016). We also observed that Bacteroidetes (39.4 vs. $15.2 \%$ ) and Fusobacteria (30.4 vs. 9.5\%) were the only 2 phyla that were differentially more abundant in metritic cows than in healthy cows. At the genus level, Fusobacterium (29.8 vs. 11.8\%), Bacteroides (21.4 vs. $7.0 \%$ ), and Porphyromonas (16.9 vs. $5.3 \%$ ) were the most prevalent genera more abundant in metritic cows than in healthy cows (Figure 3C,D). Similar to our original data (Jeon et al., 2015), this large data set supports the involvement of other less prevalent $(<5 \%)$ genera, such as Peptostreptococcus, Filifactor, Prevotella, Helcococcus, Peptoniphilus, and Arcanobacterium. Interestingly, with the exception of Prevotella, these other genera are gram-positive. Finally, we determined Spearman correlations among these main bacteria and propose an updated picture of the interactions among
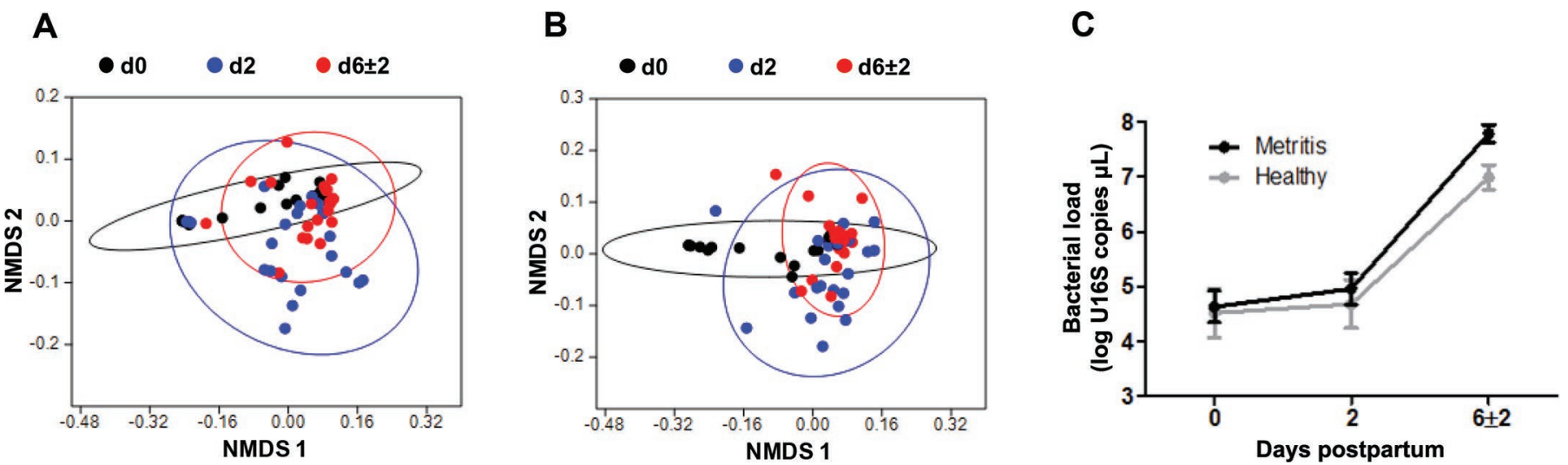

Figure 1. Non-metric multidimensional scaling (NMDS) of uterine bacterial communities based on Bray-Curtis dissimilarity at the (A) phylum and (B) genus levels, and (C) quantitative PCR of total bacteria from calving (d 0) until $6 \pm 2$ d postpartum (DPP). Error bars indicate SEM. 
the main bacteria associated with metritis at the time of diagnosis (Figure 4).

It is worth noting that many of these pathogens have never been recognized by conventional culture studies, and therefore, are potential emerging uterine pathogens. Indeed, we investigated the leads from our previous $16 \mathrm{~S}$ rRNA gene sequencing studies using quantitative PCR, and confirmed that metritic cows had greater copy numbers of Bacteroides pyogenes and Helcococcus ovis, which were the main species from the genera Bacteroides and Helcococcus, respectively (Jeon et al., 2016; Cunha et al., 2018).

Healthy cows maintain a much more heterogeneous and rich uterine microbiome compared with metritic cows (Jeon et al., 2015, 2016; Figure 2A-F; Figure 3A,B). We observed that Proteobacteria and Tenericutes were numerically more abundant in healthy cows, and that several genera were associated with uterine health including Escherichia, Serratia, Mycoplasma, Ureaplasma, and Ruminococcus, among others (Jeon et al., 2015). Herein, we confirmed that the uterine microbiome of healthy cows is more complex than that of metritic cows. Proteobacteria, Tenericutes, and several other phyla were more abundant in healthy cows than in metritic cows (Figure 3C). At the genus level, Tralbulsiella, Escherichia, Mycoplasma, and Ureaplasma, among several others, were more abundant in healthy cows than in metritic cows (Figure 3D). Although some genera reported herein as being associated with uterine health, such as Tralbulsiella, differ from those in our first report, others were confirmed, such as Escherichia, Mycoplasma, Ureaplasma, and Ruminococcus (Jeon et al., 2015). It is worth mentioning that higher relative abundance of several genera in healthy cows is likely a result of maintaining a heterogeneous, diverse microbiome that is not overtaken by Fusobacteria and Bacteroidetes, as most of these genera are not found in all healthy cows, with the exception of Escherichia, Mycoplasma, and Ureaplasma, and have low prevalence $(\leq 7 \%)$ in healthy cows. In 2017 , we showed that cows that remain healthy and cows that develop metritis had sub-communities of bacteria that led to the development of metritis and sub-communities that were associated with uterine health (Jeon et al., 2017). However, in cows that develop metritis, there is a dysbiosis in the microbial community in which bacteria such as $\mathrm{Fu}$ -
A

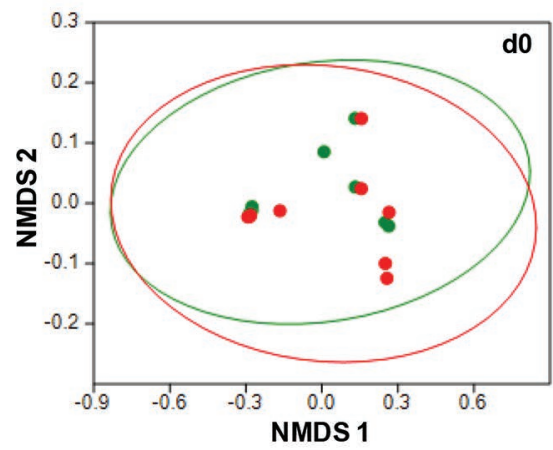

D

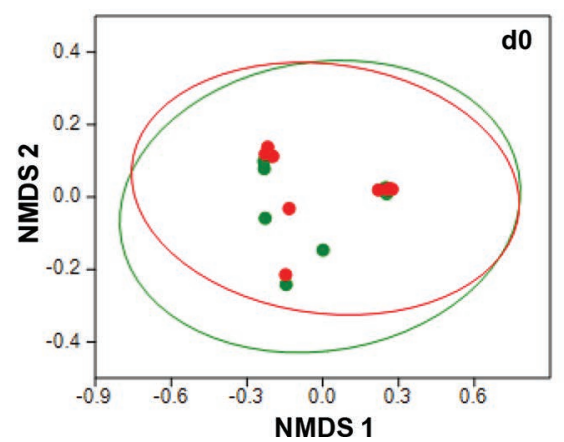

B

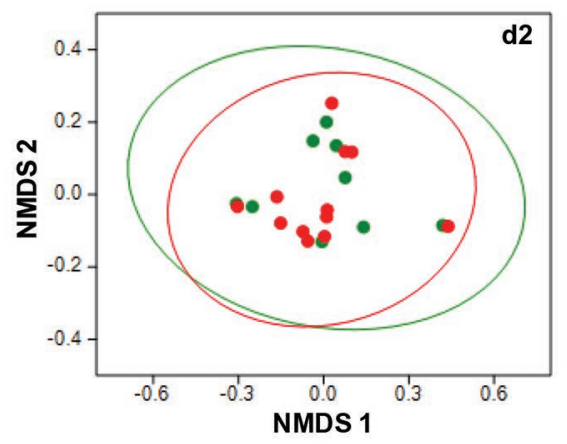

E

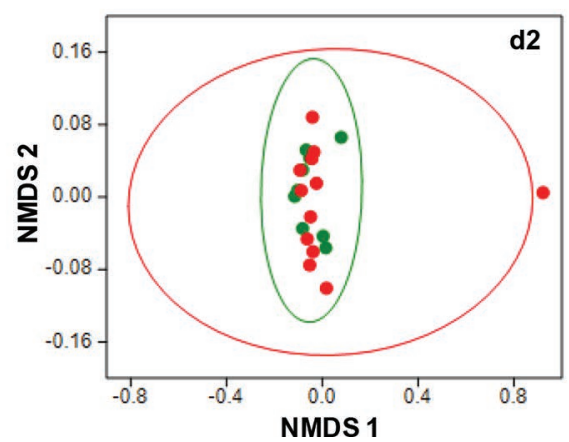

C

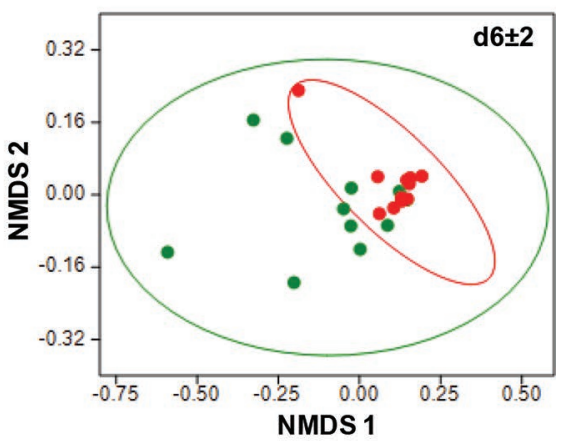

$\mathbf{F}$

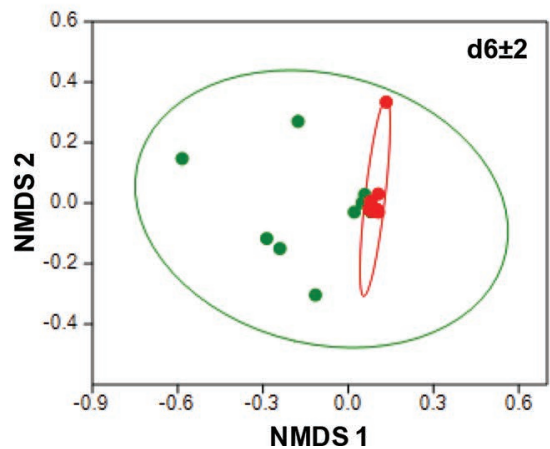

Figure 2. Non-metric multidimensional scaling (NMDS) of uterine bacterial communities based on Bray-Curtis dissimilarity at the (A, B, C) phylum and (D, E, F) genus levels from calving (d 0) until $6 \pm 2$ d postpartum (DPP). Metritis d $0=9$ cows; d $2=12$ cows; d $6 \pm 2=11$ cows; no metritis d $0=8$ cows; d $2=9$ cows; d $6 \pm 2=11$ cows. 
A

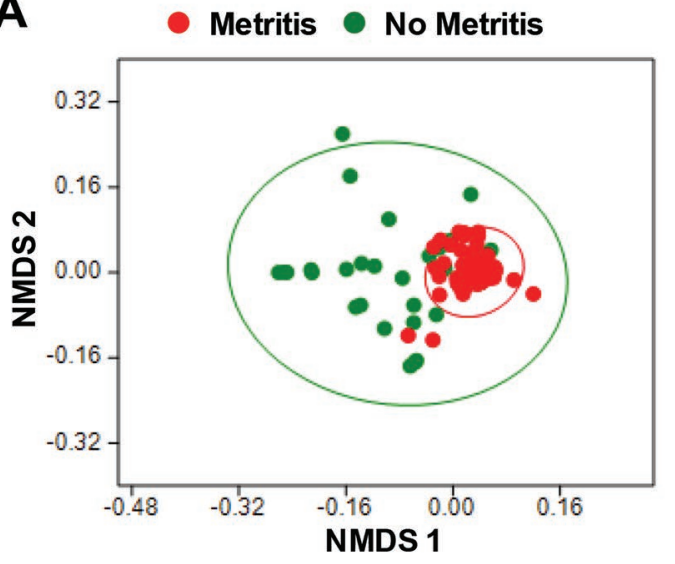

C

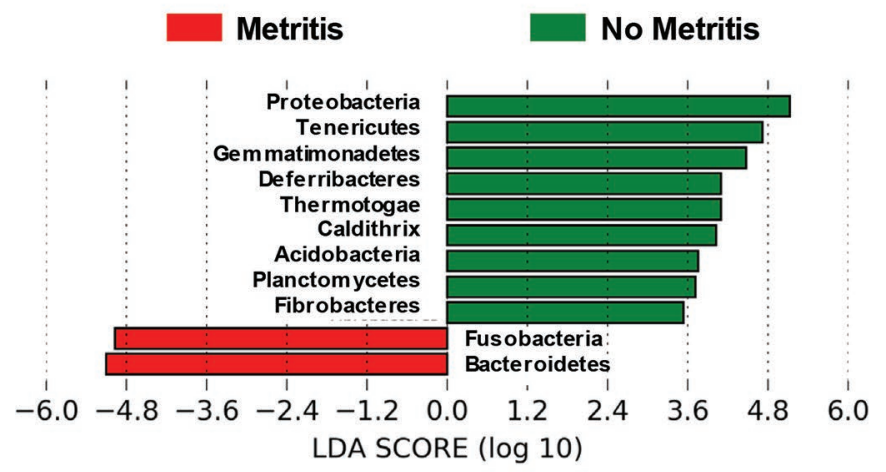

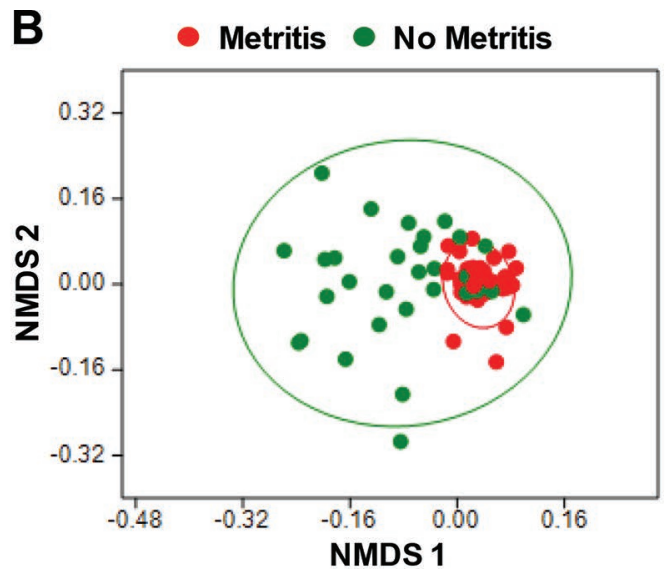

D

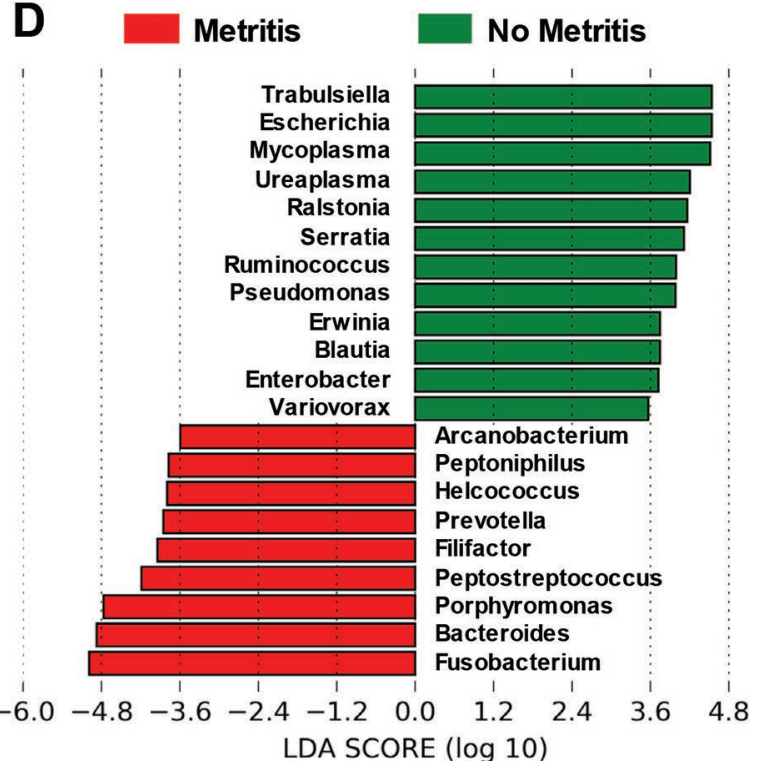

Figure 3. Non-metric multidimensional scaling (NMDS) of uterine bacterial communities based on Bray-Curtis dissimilarity at the (A) phylum and (B) genus levels, and linear discriminant analysis (LDA) effect size at the (C) phylum and (D) genus levels at the time of metritis diagnosis. Metritis $=80$ cows; no metritis $=31$ cows.

sobacterium, Bacteroides, and Porphyromonas overtake others such as Escherichia, Mycoplasma, Ureaplasma, and Ruminococcus, hence decreasing microbial richness (Jeon et al., 2015, 2016; Sicsic et al., 2018; Galvão et al., 2019). Therefore, studies of the microbiome show that all cows have all the uterine pathogens; however, cows that remain healthy are better able to prevent the shift toward greater abundance of Fusobacteria and Bacteroidetes. This is illustrated in Figure 5. In that sense, bacterial resistance seems to be an important component of maintaining uterine health. Nonetheless, total bacterial load and the load of specific pathogens also increase postpartum (Bicalho et al., 2017b), which indicates a component of bacterial tolerance in maintaining uterine health. At this point, it is unclear what factors contribute the most to differences in bacterial resistance and tolerance and the dysbiosis that leads to uterine disease. It is likely that major risk factors such as parity, dystocia, twins, stillbirth, retained placenta, and metabolic stress affect bacterial resistance and tolerance. Indeed, cows with dystocia, twins, stillbirth, and retained placenta have been shown to have decreased bacterial resistance postpartum (Bicalho et al., 2010, 2017b).

Given the fact that healthy cows have an abundance of bacteria in the uterus, including pathogenic bacteria, what is the usefulness to antibiotic treatment for cows with uterine disease? We just showed that treating cows for metritis with ceftiofur increased cure rates, did not decrease culling in the first $60 \mathrm{~d}$ postpartum, but increased milk yield and fertility up to $300 \mathrm{~d}$ postpartum (Oliveira et al., 2019). Others have shown that treating 


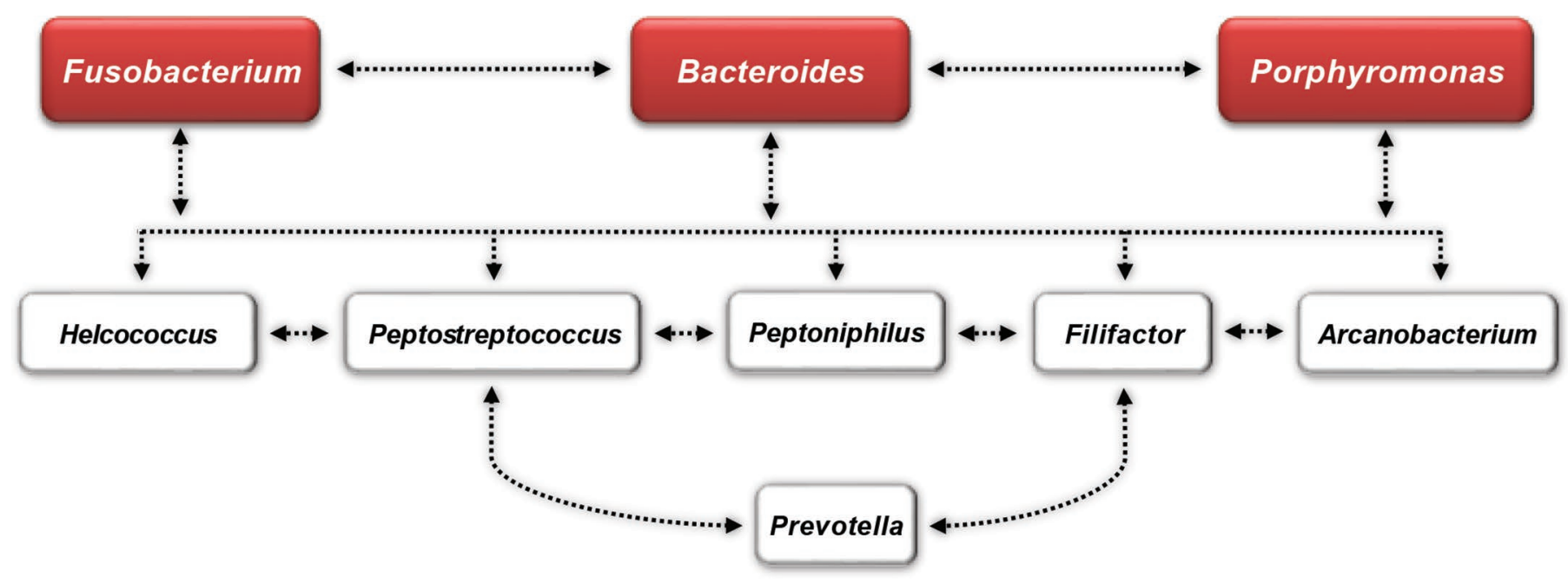

Figure 4. Microbial interactions associated with metritis. Bacteria in red boxes are the most prevalent $(>15 \%)$ genera that were more abundant in the metritis group in the linear discriminant analysis effect size analysis. Bacteria in the white boxes were more abundant in the metritis group but had low prevalence $(<5 \%)$. Arrows indicate positive correlations $(P \leq 0.02)$.

cows with clinical endometritis with cephapirin sodium also improves fertility (LeBlanc et al., 2002b; DenisRobichaud and Dubuc, 2015); therefore, treatment of cows with uterine diseases with antibiotics should take into account welfare, economics, and antibiotic resistance considerations.

Interestingly, we saw that a sub-community of uterine pathogens that included Fusobacterium, Bacteroides, and Porphyromonas was also present in blood shortly after calving, which indicate that a hematogenous route of uterine infection is feasible in cows (Jeon et al., 2017). Therefore, uterine pathogens could be transferred to the uterus shortly after calving or even before calving. Indeed, a study showed that bacterial translocation to extra-intestinal sites was more common in late gestation and shortly after parturition than in the nonpregnant state or in early or mid lactation mice (Perez et al., 2007). Furthermore, recent studies using fluorescence in situ hybridization reported the presence of F. necrophorum, Por. levii, and T. pyogenes within the endometrium, on the endometrial surface, and in the caruncular stroma of pregnant cows, which indicates that bacterial pathogens could be in the uterus even before parturition (Karstrup et al., 2017). Using massive parallel sequencing, the same study reported that Porphyromonadaceae was the most prevalent family of bacteria (Karstrup et al., 2017). Interestingly, the presence of bacteria in the uterus prepartum was not associated with inflammation, which indicates that there is increased bacterial resistance and tolerance during pregnancy. This is likely a result of inadequate conditions for bacterial proliferation, such as an in- tact endometrium, adequate oxygen perfusion, lack of substrate for bacterial growth, and a switch from a T-helper (Th)1 to a Th2 response during pregnancy. Nonetheless, bacterial proliferation and inflammation (placentitis) is likely a cause of pregnancy loss in cattle. During routine pregnancy diagnosis using ultrasonography between 60 and $90 \mathrm{~d}$ after breeding, the authors (K. N. Galvão and R. C. Bicalho) have observed several cows with echogenic fluid, characteristic of pus, between the endometrium and the chorioallantois. These changes invariably led to pregnancy loss. Unfortunately, the causative agents of these early-gestation abortions are not usually investigated. In contrast, mid- to lategestation abortions are investigated, and the causative agents are varied and include uterine pathogens such as T. pyogenes (Anderson, 2007). Pregnancy loss due to placentitis is a well-documented problem in mares and is believed to be caused by ascending infection (Macpherson and Bailey, 2008). In cows, deep-seated infections or hematogenous translocation may be the most likely cause. Some pathogens are more likely to be deep-seated such as Tritrichomonas foetus and Campylobacter fetus, which spread venereally, whereas others are more likely to translocate from blood such as Brucella abortus and Leptospira spp., which transmit by direct contact. Regarding uterine pathogens such as T. pyogenes, both routes may be possible. Moore et al. (2017) also observed that the uterine endometrium from virgin heifers and pregnant uteri harbored a microbiome, in which Firmicutes and Bacteroidetes were the most prevalent phyla. Although bacteria could already be in the uterus or arrive there shortly after 
calving through the hematogenous route, it is likely that ascending contamination from the vagina or the environment through the vagina is also possible.

Machado et al. (2012) explored the uterine microbiome of cows with clinical endometritis using pyrosequencing of the 16S rRNA gene, and observed that cows with clinical endometritis had higher relative abundance of Bacteroidetes, particularly Bacteroides; Tenericutes, particularly Ureaplasma; Fusobacteria, particularly Fusobacterium; and Actinobacteria, particularly Trueperella, whereas healthy cows had higher relative abundance of Firmicutes, particularly Geobacillus. These data showed that the uterine microbiome of cows with clinical endometritis was very similar to that of cows with metritis, except for the higher relative abundance of Tenericutes in cows with clinical endometritis. Higher relative abundance of Bacteroidetes and Fusobacterium in cows that developed clinical endometritis was later confirmed by others (Wang et al., 2018; Miranda-CasoLuengo et al., 2019).

The advent of metagenomic tools such as $16 \mathrm{~S}$ rRNA gene sequencing, allowed researchers to characterize the bacterial sub-communities associated with uterine health and disease in cows. Whereas culture-dependent studies pointed to $T$. pyogenes and E. coli as the most prevalent uterine pathogens, metagenomic studies

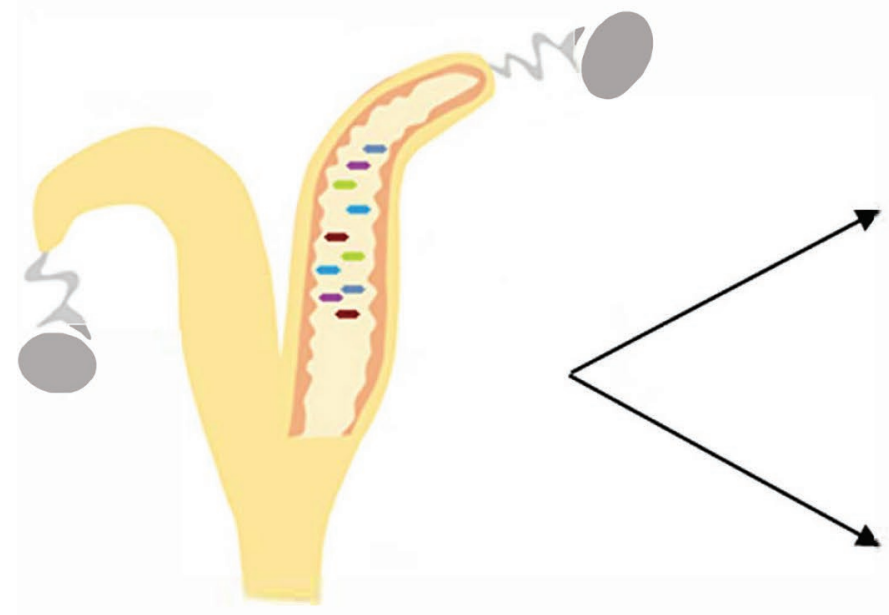

Healthy Uterus

Bacteroides

Fusobacteria

Proteobacteria

Tenericutes

Firmicutes
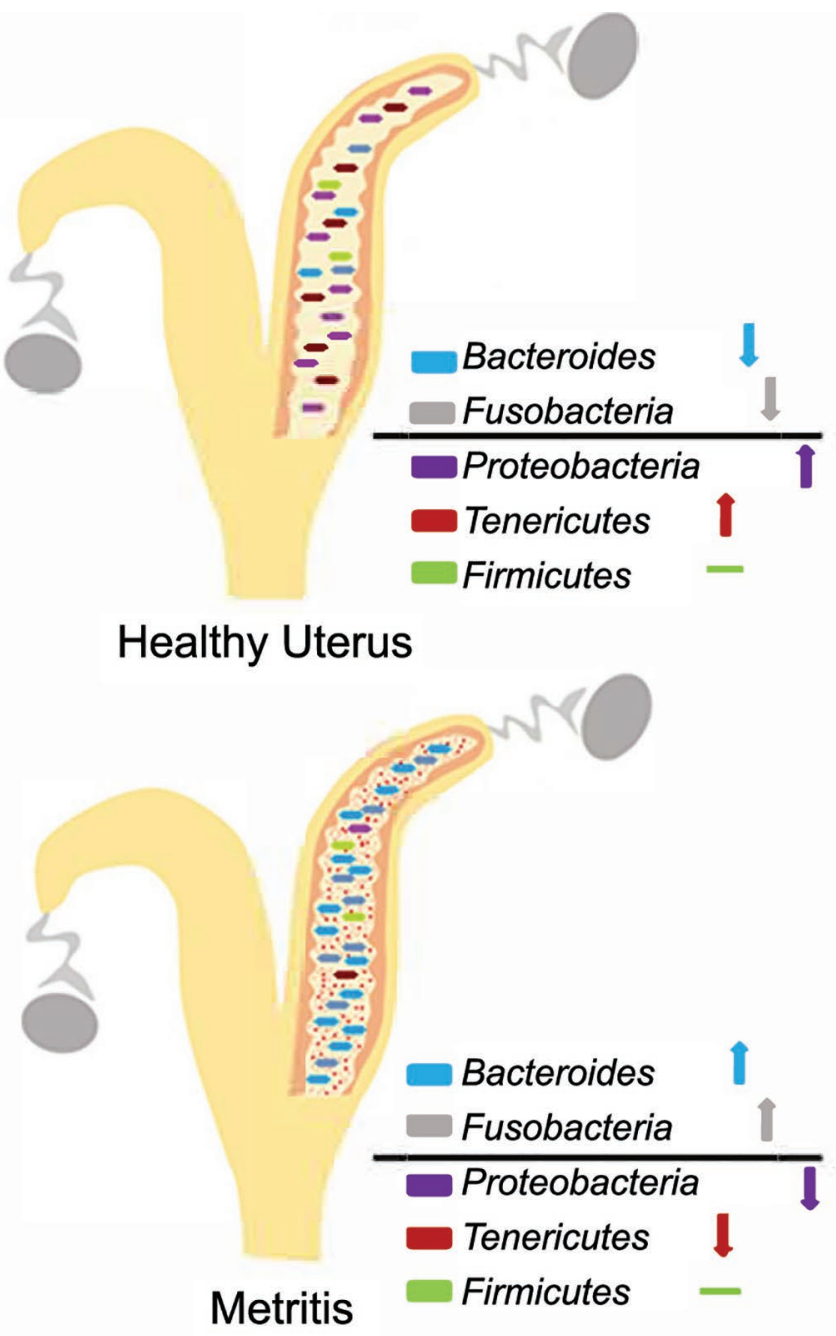

0-2 DPP

\section{4-14 DPP}

Figure 5. Microbial shift at the phylum level from calving until establishment of metritis in dairy cows. Arrows following phylum names indicate relative abundance comparison among cows with metritis and healthy cows; a dash indicates no difference in relative abundance. DPP $=$ days postpartum. 
showed that Fusobacterium (particularly F. necrophorum), Porphyromonas (particularly Por. levii), and Bacteroides (particularly B. pyogenes) are the most prevalent bacteria in the uterus of cows with uterine disease. Metagenomic studies allowed us to recognize emerging pathogens such as B. pyogenes and $H$. ovis. Additionally, metagenomic studies allowed us to better characterize the complex interactions among bacteria, even in tissues where culture is usually unproductive such as the pregnant uterus and blood. Hence, we now have a much better understanding of the major bacteria associated with metritis and clinical endometritis, which is the first step to studying the microbiome. The next step is to perform integrative studies that involve whole-metagenome sequencing and metabolome and immune function analysis to better understand the complex interactions that lead to immunosuppression, microbial dysbiosis, and disease establishment, so we can work on establishing causation rather than just associations (Segata et al., 2013; Aguiar-Pulido et al., 2016; Schmidt et al., 2018). Additionally, understanding the web of causation will allow development of new prebiotics, probiotics, and therapeutics to prevent and treat uterine disease in dairy cows. This leap has been taken on the human side with the integrative human microbiome project (iHMP; https://www.hmpdacc .org/ihmp/); a similar approach in the animal side is needed to advance our understanding of factors that affect animal health and disease.

\section{CONCLUSIONS}

Cows had bacteria in the uterus even before calving, they had an established uterine microbiome within 20 min of calving, and the microbiome structure was identical between cows that developed metritis and healthy cows up until $2 \mathrm{~d}$ postpartum, after which the bacterial composition of cows that developed metritis deviated in favor of greater relative abundance of Bacteroidetes and Fusobacteria and lesser relative abundance of Proteobacteria and Tenericutes. The shift in the uterine microbiome in cows that developed metritis is characterized by a loss of heterogeneity and a decrease in bacterial richness. At the genus level, Bacteroides, Porphyromonas, and Fusobacterium seem to act synergistically to cause metritis. At the species level, we identified $B$. pyogenes, Por. levii, and $H$. ovis as potential emerging uterine pathogens, and confirmed $F$. necrophorum as one of the species mainly associated with uterine disease. We also observed that failure to cure metritis was associated with an increase in the relative abundance of Bacteroides, Porphyromonas, and Fusobacterium, and a further decrease in bacterial diversity. The structure of the uterine microbiome was similar between cows that did and did not have a fever at the time of diagnosis. Finally, we showed that the hematogenous route is a viable route of uterine infection with uterine pathogens such as Bacteroides, Porphyromonas, and Fusobacteri$u m$. Herein, we propose that metritis is associated with dysbiosis of the uterine microbiota characterized by decreased richness, and an increase in Bacteroidetes and Fusobacteria, particularly Bacteroides, Porphyromonas, and Fusobacterium.

\section{ACKNOWLEDGMENTS}

Microbiome data presented in this review were generated with the support of grants from the USDANIFA-CRIS program (National Institute for Food and Agriculture, Washington, DC; Accession Number: 1002880) and the USDA-NIFA-AFRI program (Accession Number: 1008863). We extend our gratitude to Segundo Casaro (Department of Large Animal Clinical Sciences, College of Veterinary Medicine, University of Florida, Gainesville) for his contribution in creating Figure 5.

\section{REFERENCES}

Aguiar-Pulido, V., W. Huang, V. Suarez-Ulloa, T. Cickovski, K. Mathee, and G. Narasimhan. 2016. Metagenomics, metatranscriptomics, and metabolomics approaches for microbiome analysis. Evol. Bioinform. Online 12(Suppl. 1):5-16.

Anderson, M. L. 2007. Infectious causes of bovine abortion during mid- to late gestation. Theriogenology 68:474-486.

Bicalho, M. L., V. S. Machado, C. H. Higgins, F. S. Lima, and R. C. Bicalho. 2017a. Genetic and functional analysis of the bovine uterine microbiota. Part I: Metritis versus healthy cows. J. Dairy Sci. 100:3850-3862.

Bicalho, M. L., V. S. Machado, G. Oikonomou, R. O. Gilbert, and R. C. Bicalho. 2012. Association between virulence factors of Escherichia coli, Fusobacterium necrophorum, and Arcanobacterium pyogenes and uterine diseases of dairy cows. Vet. Microbiol. 157:125-131.

Bicalho, M. L., T. Santin, M. X. Rodrigues, C. E. Marques, S. F. Lima, and R. C. Bicalho. 2017b. Dynamics of the microbiota found in the vaginas of dairy cows during the transition period: Associations with uterine diseases and reproductive outcome. J. Dairy Sci. 100:3043-3058.

Bicalho, R. C., V. S. Machado, M. L. Bicalho, R. O. Gilbert, A. G. Teixeira, L. S. Caixeta, and L. V. Pereira. 2010. Molecular and epidemiological characterization of bovine intrauterine Escherichia coli. J. Dairy Sci. 93:5818-5830.

Bonnett, B. N., S. W. Martin, V. P. Gannon, R. B. Miller, and W. G. Etherington. 1991. Endometrial biopsy in Holstein-Friesian dairy cows. III. Bacteriological analysis and correlations with histological findings. Can. J. Vet. Res. 55:168-173.

Bonnett, B. N., S. W. Martin, and A. H. Meek. 1993. Associations of clinical findings, bacteriological and histological results of endometrial biopsy with reproductive performance of postpartum dairy cows. Prev. Vet. Med. 15:205-220.

Cai, T. Q., P. G. Weston, L. A. Lund, B. Brodie, D. J. McKenna, and W. C. Wagner. 1994. Association between neutrophil functions and periparturient disorders in cows. Am. J. Vet. Res. 55:934-943.

Chakravorty, S., D. Helb, M. Burday, N. Connell, and D. Alland. 2007. A detailed analysis of $16 \mathrm{~S}$ ribosomal RNA gene segments 
for the diagnosis of pathogenic bacteria. J. Microbiol. Methods 69:330-339.

Cunha, F., S. J. Jeon, R. Daetz, A. Vieira-Neto, J. Laporta, K. C. Jeong, A. F. Barbet, C. A. Risco, and K. N. Galvão. 2018. Quantifying known and emerging uterine pathogens, and evaluating their association with metritis and fever in dairy cows. Theriogenology 114:25-33.

de Boer, M., B. M. Buddle, C. Heuer, H. Hussein, T. Zheng, S. J. LeBlanc, and S. McDougall. 2015. Associations between intrauterine bacterial infection, reproductive tract inflammation, and reproductive performance in pasture-based dairy cows. Theriogenology 83:1514-1524.

Denis-Robichaud, J., and J. Dubuc. 2015. Randomized clinical trial of intrauterine cephapirin infusion in dairy cows for the treatment of purulent vaginal discharge and cytological endometritis. J. Dairy Sci. 98:6856-6864.

Dohmen, M. J., K. Joop, A. Sturk, P. E. Bols, and J. A. Lohuis. 2000. Relationship between intra-uterine bacterial contamination, endotoxin levels and the development of endometritis in postpartum cows with dystocia or retained placenta. Theriogenology 54:1019 1032.

Dohmen, M. J., J. A. Lohuis, Gy. Huszenicza, P. Nagy, and M. Gacs. 1995. The relationship between bacteriological and clinical findings in cows with subacute/chronic endometritis. Theriogenology 43:1379-1388.

Drillich, M., O. Beetz, A. Pfützner, M. Sabin, H. J. Sabin, P. Kutzer, H. Nattermann, and W. Heuwieser. 2001. Evaluation of a systemic antibiotic treatment of toxic puerperal metritis in dairy cows. J. Dairy Sci. 84:2010-2017.

Elliott, L., K. J. McMahon, H. T. Gier, and G. B. Marion. 1968. Uterus of the cow after parturition: bacterial content. Am. J. Vet. Res. 29:77-81.

Farin, P. W., L. Ball, J. D. Olson, R. G. Mortimer, R. L. Jones, W S. Adney, and A. E. McChesney. 1989. Effect of Actinomyces pyogenes and gram-negative anaerobic bacteria on the development of bovine pyometra. Theriogenology 31:979-989.

Galvão, K. N. 2012. Postpartum uterine diseases in dairy cows. Anim. Reprod. 9:290-296.

Galvão, K. N., C. H. Higgins, M. Zinicola, S. J. Jeon, H. Korzec, and R. C. Bicalho. 2019. Effect of pegbovigrastim administration on the microbiome found in the vagina of cows postpartum. J. Dairy Sci. 102:3439-3451.

Galvão, K. N., and J. E. Santos. 2014. Recent advances in the immunology and uterine microbiology of healthy cows and cows that develop uterine disease. Turk. J. Vet. Anim. Sci. 38:577-588.

Gilbert, R. O., and N. R. Santos. 2016. Dynamics of postpartum endometrial cytology and bacteriology and their relationship to fertility in dairy cows. Theriogenology 85:1367-1374.

Gonzalez-Pena, D., H. Jeong, T. M. Goncalves, P. J. Pinedo, J. E. P. Santos, G. M. Schuenemann, G. J. M. Rosa, R. O. Gilbert, R. C. Bicalho, R. Chebel, K. N. Galvão, C. M. Seabury, W. W. Thatcher, and S. L. Rodriguez Zas. 2016. Genetic parameters of early lactation diseases in dairy cattle. J. Dairy Sci. 99(E. Suppl. 1):173. (Abstr.)

Goshen, T., and N. Y. Shpigel. 2006. Evaluation of intrauterine antibiotic treatment of clinical metritis and retained fetal membranes in dairy cows. Theriogenology 66:2210-2218.

Griffin, J. F., P. J. Hartigan, and W. R. Nunn. 1974. Non-specific uterine infection and bovine fertility. I. Infection patterns and endometritis during the first seven weeks post-partum. Theriogenology 1:91-106.

Huszenicza, Gy., M. Fodor, M. Gacs, M. Kulcsar, M. J. Dohmen, M. Vamos, L. Porkolab, T. Kegl, J. Bartyik, J. A. Lohuis, Sz. Janosi, and G. Szita. 1999. Uterine bacteriology, resumption of cyclic ovarian activity and fertility in postpartum cows kept in large-scale dairy herds. Reprod. Domest. Anim. 34:237-245.

Jeon, S. J., F. Cunha, X. Ma, N. Martinez, A. Vieira-Neto, R. Daetz, R. C. Bicalho, S. Lima, J. E. Santos, K. C. Jeong, and K. N. Galvão. 2016. Uterine microbiota and immune parameters associated with fever in dairy cows with metritis. PLoS One 11:e0165740.
Jeon, S. J., F. Cunha, A. Vieira-Neto, R. C. Bicalho, S. Lima, M. L. Bicalho, and K. N. Galvão. 2017. Blood as a route of transmission of uterine pathogens from the gut to the uterus in cows. Microbiome 5:109.

Jeon, S. J., F. S. Lima, A. Vieira-Neto, V. S. Machado, S. F. Lima, R. C. Bicalho, J. E. P. Santos, and K. N. Galvão. 2018. Shift of uterine microbiota associated with antibiotic treatment and cure of metritis in dairy cows. Vet. Microbiol. 214:132-139.

Jeon, S. J., A. Vieira-Neto, M. Gobikrushanth, R. Daetz, R. D. Mingoti, A. C. Parize, S. L. de Freitas, A. N. da Costa, R. C. Bicalho, S. Lima, K. C. Jeong, and K. N. Galvão. 2015. Uterine microbiota progression from calving until establishment of metritis in dairy cows. Appl. Environ. Microbiol. 81:6324-6332.

Kaeberlein, T., K. Lewis, and S. S. Epstein. 2002. Isolating "uncultivable" microorganisms in pure culture in a simulated natural environment. Science 296:1127-1129.

Karstrup, C. C., K. Klitgaard, T. K. Jensen, J. S. Agerholm, and H. G. Pedersen. 2017. Presence of bacteria in the endometrium and placentomes of pregnant cows. Theriogenology 99:41-47.

Kehrli, M. E. Jr., and J. P. Goff. 1989. Periparturient hypocalcemia in cows: Effects on peripheral blood neutrophil and lymphocyte function. J. Dairy Sci. 72:1188-1196.

Knudsen, L. R., C. C. Karstrup, H. G. Pedersen, O. Angen, J. S. Agerholm, E. L. Rasmussen, T. K. Jensen, and K. Klitgaard. 2016. An investigation of the microbiota in uterine flush samples and endometrial biopsies from dairy cows during the first 7 weeks postpartum. Theriogenology 86:642-650.

Lamming, G. E., A. Seaman, and M. Woodbine. 1955. Relationship of ovarian hormones to the pyogenic effect of Corynebacterium pyogenes. Nature 175:126.

LeBlanc, S. J. 2008. Postpartum uterine disease and dairy herd reproductive performance: A review. Vet. J. 176:102-114.

LeBlanc, S. J., T. F. Duffield, K. E. Leslie, K. G. Bateman, G. P. Keefe, J. S. Walton, and W. H. Johnson. 2002a. Defining and diagnosing postpartum clinical endometritis and its impact on reproductive performance in dairy cows. J. Dairy Sci. 85:2223-2236.

LeBlanc, S. J., T. F. Duffield, K. E. Leslie, K. G. Bateman, G. P. Keefe, J. S. Walton, and W. H. Johnson. 2002b. The effect of treatment of clinical endometritis on reproductive performance in dairy cows. J. Dairy Sci. 85:2237-2249.

Lima, F. S., L. F. Greco, R. S. Bisinotto, E. S. Ribeiro, N. M. Martinez, W. W. Thatcher, J. E. Santos, M. K. Reinhard, and K. N. Galvão. 2015. Effects of intrauterine infusion of Trueperella pyogenes on endometrial mRNA expression of proinflammatory cytokines and luteolytic cascade genes and their association with luteal life span in dairy cows. Theriogenology 84:1263-1272.

Machado, V. S., G. Oikonomou, M. L. Bicalho, W. A. Knauer, R. Gilbert, and R. C. Bicalho. 2012. Investigation of postpartum dairy cows' uterine microbial diversity using metagenomic pyrosequencing of the 16S rRNA gene. Vet. Microbiol. 159:460-469.

Macpherson, M. L., and C. S. Bailey. 2008. A clinical approach to managing the mare with placentitis. Theriogenology 70:435-440

Miranda-CasoLuengo, R., J. Lu, E. J. Williams, A. A. Miranda-CasoLuengo,, S. D. Carrington, A. C. O. Evans, and W. G. Meijer. 2019. Delayed differentiation of vaginal and uterine microbiomes in dairy cows developing postpartum endometritis. PLoS One 14:e0200974.

Mizrahi-Man, O., E. R. Davenport, and Y. Gilad. 2013. Taxonomic classification of bacterial 16S rRNA genes using short sequencing reads: Evaluation of effective study designs. PLoS One 8:e53608.

Moore, S. G., A. C. Ericsson, S. E. Poock, P. Melendez, and M. C. Lucy. 2017. Hot topic: 16S rRNA gene sequencing reveals the microbiome of the virgin and pregnant bovine uterus. J. Dairy Sci. 100:4953-4960.

Noakes, D. E., L. Wallace, and G. R. Smith. 1991. Bacterial flora of the uterus of cows after calving on two hygienically contrasting farms. Vet. Rec. 128:440-442.

Oliveira, E., F. Cunha, R. Daetz, R. Chebel, C. Risco, J. Santos, K. Jeong, and K. N. Galvão. 2019. Using chitosan microparticles to treat metritis in lactating dairy cows. J. Dairy Sci. 102(Suppl. 1):286. (Abstr.) 
Olson, J. D., L. Ball, R. G. Mortimer, P. W. Farin, W. S. Adney, and E. M. Huffman. 1984. Aspects of bacteriology and endocrinology of cows with pyometra and retained fetal membranes. Am. J. Vet. Res. 45:2251-2255.

Perez, P. F., J. Dore, M. Leclerc, F. Levenez, J. Benyacoub, P. Serrant, I. SeguraRoggero, E. J. Schiffrin, and A. Donnet-Hughes. 2007. Bacterial imprinting of the neonatal immune system: lessons from maternal cells? Pediatrics 119:e724-e732.

Piersanti, R. L., R. Zimpel, P. C. C. Molinari, M. J. Dickson, Z. Ma, K. C. Jeong, J. E. P. Santos, I. M. Sheldon, and J. J. Bromfield. 2019. A model of clinical endometritis in Holstein heifers using pathogenic Escherichia coli and Trueperella pyogenes. J. Dairy Sci. 102:2686-2697.

Ruder, C. A., R. G. Sasser, R. J. Williams, J. K. Ely, R. C. Bull, and J. E. Butler. 1981. Uterine infections in the postpartum cow. II. Possible synergistic effect of Fusobacterium necrophorum and Corynebacterium pyogenes. Theriogenology 15:573-580.

Santos, T. M., R. O. Gilbert, and R. C. Bicalho. 2011. Metagenomic analysis of the uterine bacterial microbiota in healthy and metritic postpartum dairy cows. J. Dairy Sci. 94:291-302.

Schmidt, T. S. B., J. Raes, and P. Bork. 2018. The human gut microbiome: From association to modulation. Cell 172:1198-1215.

Schuenemann, G. M., I. Nieto, S. Bas, K. N. Galvão, and J. Workman. 2011. Dairy calving management: Effect of perineal hygiene scores on metritis. J. Dairy Sci. 94:744.

Segata, N., D. Boernigen, T. L. Tickle, X. C. Morgan, W. S. Garrett, and C. Huttenhower. 2013. Computational metaomics for microbial community studies. Mol. Syst. Biol. 9:666-680.

Segata, N., J. Izard, L. Waldron, D. Gevers, L. Miropolsky, W. S. Garrett, and C. Huttenhower. 2011. Metagenomic biomarker discovery and explanation. Genome Biol. 12:R60.

Sheldon, I. M., J. Cronin, L. Goetze, G. Donofrio, and H. J. Schuberth. 2009. Defining postpartum uterine disease and the mechanisms of infection and immunity in the female reproductive tract in cattle. Biol. Reprod. 81:1025-1032.

Sheldon, I. M., J. G. Cronin, and J. J. Bromfield. 2019. Tolerance and innate immunity shape the development of postpartum uterine disease and the impact of endometritis in dairy cattle. Annu. Rev. Anim. Biosci. 7:361-384.

Sheldon, I. M., and H. Dobson. 2004. Postpartum uterine health in cattle. Anim. Reprod. Sci. 82-83:295-306.
Sheldon, I. M., G. S. Lewis, S. LeBlanc, and R. O. Gilbert. 2006 Defining postpartum uterine disease in cattle. Theriogenology 65:1516-1530.

Sheldon, I. M., D. E. Noakes, A. N. Rycroft, D. U. Pfeiffer, and H. Dobson. 2002. Influence of uterine bacterial contamination after parturition on ovarian dominant follicle selection and follicle growth and function in cattle. Reproduction 123:837-845.

Sheldon, I. M., A. N. Rycroft, B. Dogan, M. Craven, J. J. Bromfield, A. Chandler, M. H. Roberts, S. B. Price, R. O. Gilbert, and K. W. Simpson. 2010. Specific strains of Escherichia coli are pathogenic for the endometrium of cattle and cause pelvic inflammatory disease in cattle and mice. PLoS One 5:e9192.

Sicsic, R., T. Goshen, R. Dutta, N. Kedem-Vaanunu, V. KaplanShabtai, Z. Pasternak, Y. Gottlieb, N. Y. Shpigel, and T. Raz. 2018. Microbial communities and inflammatory response in the endometrium differ between normal and metritic dairy cows at 5-10 days post-partum. Vet. Res. 49:77.

Studer, E., and D. A. Morrow. 1978. Postpartum evaluation of bovine reproductive potential: Comparison of findings from genital tract examination per rectum, uterine culture, and endometrial biopsy. J. Am. Vet. Med. Assoc. 172:489-494.

Tchesnokova, V., P. Aprikian, D. Kisiela, S. Gowey, N. Korotkova, W. Thomas, and E. Sokurenko. 2011. Type 1 fimbrial adhesin FimH elicits an immune response that enhances cell adhesion of Escherichia coli. Infect. Immun. 79:3895-3904.

Wang, M. L., M. C. Liu, J. Xu, L. G. An, J. F. Wang, and Y. H. Zhu. 2018. Uterine microbiota of dairy cows with clinical and subclinical endometritis. Front. Microbiol. 9:2691.

Williams, E. J., D. P. Fischer, D. U. Pfeiffer, G. C. England, D. E. Noakes, H. Dobson, and I. M. Sheldon. 2005. Clinical evaluation of postpartum vaginal mucus reflects uterine bacterial infection and the immune response in cattle. Theriogenology 63:102-117.

\section{ORCIDS}

Klibs N. Galvão (®) https://orcid.org/0000-0002-6683-4476 Rodrigo C. Bicalho ㄴ https://orcid.org/0000-0003-4680-1981 